\title{
Regenerative Growth of Corticospinal Tract Axons via the Ventral Column after Spinal Cord Injury in Mice
}

\author{
Oswald Steward, ${ }^{1,2}$ Binhai Zheng, ${ }^{3}$ Marc Tessier-Lavigne, ${ }^{4}$ Maura Hofstadter, ${ }^{1}$ Kelli Sharp, ${ }^{1}$ and Kelly Matsudaira Yee ${ }^{1}$ \\ ${ }^{1}$ Reeve-Irvine Research Center and ${ }^{2}$ Departments of Anatomy and Neurobiology, and Neurobiology and Behavior, University of California, Irvine, Irvine, \\ California 92697-4292, ${ }^{3}$ Department of Neurosciences, University of California at San Diego, La Jolla, California 92093, and ${ }^{4}$ Genentech, Inc., South San \\ Francisco, California 94080
}

Studies that have assessed regeneration of corticospinal tract (CST) axons in mice after genetic modifications or other treatments have tacitly assumed that there is little if any regeneration of CST axons in normal mice in the absence of some intervention. Here, we document a previously unrecognized capability for regenerative growth of CST axons in normal mice that involves growth past the lesion via the ventral column. Mice received dorsal hemisection injuries at thoracic level 6-7, which completely transect descending CST axons in the dorsal and dorsolateral column. Corticospinal projections were traced by injecting biotinylated dextran amine (BDA) into the sensorimotor cortex of one hemisphere either at the time of the injury or 4 weeks after injury, and mice were killed at $20-23$ or $46 \mathrm{~d}$ after injury. At 20-23 d after injury, BDA-labeled CST axons did not extend past the lesion except in one animal. By $46 \mathrm{~d}$ after injury, however, a novel population of BDA-labeled CST axons could be seen extending from the gray matter rostral to the injury into the ventral column, past the lesion, and then back into the gray matter caudal to the injury in which they formed elaborate terminal arbors. The number of axons with this highly unusual trajectory was small ( $\sim 1 \%$ of the total number of labeled CST axons rostral to the injury). The BDA-labeled axons in the ventral column were on the same side as the main tract and thus are not spared ventral CST axons (which would be contralateral to the main tract). These results indicate that normal mice have a capacity for CST regeneration that has not been appreciated previously, which has important implications in studying the effect of genetic or pharmacological manipulations on CST regeneration in mice.

Key words: spinal cord injury; corticospinal tract; axon regeneration; sprouting; motor system; mice; biotinylated dextran amine; BDA; tract tracing

\section{Introduction}

Deficits in sensory and motor function after spinal cord injury are attributable primarily to the interruption of the long ascending sensory and descending tracts of the spinal cord. These deficits could at least theoretically be alleviated if it was possible to induce regeneration of the critical long tracts. Although this has long been recognized as a highly desirable goal, efforts to induce meaningful axon regeneration after spinal cord injury have been disappointing. Damaged axons do seem to initiate a limited growth response, but regeneration is abortive in that axons do not penetrate the lesion, cross it, or grow around it through surviving bridges of white matter in significant numbers.

The failure of axonal regeneration after injury to the CNS of mammals is attributed to a number of factors, including the following: (1) the presence of a tissue environment at the lesion site that is extremely hostile to growing axons, including the presence

Received Dec. 4, 2007; revised May 20, 2008; accepted May 21, 2008.

This work was supported by National Institutes of Health Grants R01 NS047718 and N01-NS-3-2353 (0.S.), the Roman Reed Spinal Cord Injury Research Fund of California, Research for Cure, and individual donations to the Reeve-Irvine Research Center.

This article is freely available online through the J Neurosci Open Choice option.

Correspondence should be addressed to Dr. Oswald Steward, Reeve-Irvine Research Center, University of California, Irvine, 1105 Gillespie Neuroscience Research Facility, Irvine, CA 92697-4292. E-mail: osteward@uci.edu. D01:10.1523/JNEUROSCI.5372-07.2008

Copyright $\odot 2008$ Society for Neuroscience $\quad$ 0270-6474/08/286836-12\$15.00/0 of large cystic cavities (McDonald, 1999); (2) the presence of inhibitory boundaries associated with the astroglial scar (Silver and Miller, 2004; Yiu and He, 2006; Fitch and Silver, 2008); (3) the presence of inhibitory molecules in myelin, including candidates such as Nogo, myelin-associated glycoprotein (MAG), and oligodendrocyte myelin glycoprotein, that may interact with one or more neuronal receptor(s) to trigger signaling events that lead to axon growth inhibition (He and Koprivica, 2004; Schwab et al., 2006); (4) lack of an adequate inherent growth response by mature neurons (Bulsara et al., 2002; Harel and Strittmatter, 2006); and (5) lack of adequate trophic support, which may lead to retrograde death of neurons whose axons were injured (Fernandes et al., 1999; Hains et al., 2003).

In recent years, mice have been used increasingly in experiments that use genetic techniques to alter molecular pathways thought to be important for regeneration (Zheng et al., 2006). In these studies, the tacit assumption has been that there is little if any regeneration of axons in the absence of some intervention. Based on this assumption, evidence of regeneration in genetically modified mice is taken as evidence that the genetic modifications triggered regeneration that was otherwise impossible. Importantly, however, although all studies include controls, there has not been a thorough characterization of what types of axon growth actually occur in normal mice. In this context, we were surprised when our studies of the corticospinal tract (CST) after 
spinal cord injuries in mice revealed a form of regenerative growth that has not been characterized previously. Specifically, after dorsal hemisection injuries, which interrupt all normal descending CST axons that are detected by tract tracing, some CST axons extend around the lesion via surviving white matter bridges in the ventral column and form elaborate terminal arbors in segments caudal to the injury. Here, we describe this phenomenon and consider its implications for studies in mice, including those that are genetically modified.

\section{Materials and Methods}

Experimental animals. The mice described here were from the control groups from experiments that were reported previously (Steward et al., $2008)$. One was a group of C57BL/6 mice $(n=12)$ that received injections of a vehicle solution ( $83 \%$ PBS plus 17\% DMSO) beginning $\sim 5 \mathrm{~h}$ after injury and continuing on a daily basis for $14 \mathrm{~d}$. This was the control group for mice that received injections of the Nogo receptor antagonist peptide NEP1-40, as described by Li and Strittmatter (2003). The second $(n=7)$ was a group of C57BL/6 mice that were implanted peripherally with osmotic minipumps (model 2002; Alzet) containing the same vehicle solution $7 \mathrm{~d}$ after spinal cord injury. This was the control group for mice that received minipumps containing the Nogo receptor antagonist peptide NEP1-40. Substantiating evidence came from studies of the littermate wild-type controls for animals lacking Nogo-A/B that received similar lesions. The ancestors of these animals were heterozygous for the gene trap deletion of Nogo-A/B described by Kim et al. (2003) and had been intercrossed in our laboratory for two to three generations to generate Nogo-A/B knock-out and littermate wild-type controls.

Spinal cord surgical procedures. Mice were initially anesthetized with Avertin $(0.5 \mathrm{ml} / 20 \mathrm{~g})$; when supplemental anesthesia was required, onefourth of the original dose was given. Body temperature was maintained by placing mice on a water-circulating jacketed heating pad at $37 \pm$ $0.5^{\circ} \mathrm{C}$. The skin over the upper thoracic area was shaved and cleaned with a Betadyne solution. The skin was incised, and then the connective and muscle tissue were bluntly dissected to expose T6 and T7. A T6 laminectomy was completed, taking care not to damage the spinal cord during the dorsal lamina removal. A dorsal hemisection was performed at T6 either using a 30 gauge needle (in the case of the C57BL/6 mice) or Moria microknife (in the case of the wild-type controls for Nogo knock-out mice). The goal was to completely sever the dorsal (dCST) and dorsolateral CST (dlCST) by creating a lesion that completely severed the dorsal half of the spinal cord down to the central canal. To ensure that the lesion was complete laterally, the microknife or needle was passed through the dorsal part of the spinal cord several times.

Tract tracing. To trace the corticospinal tract, tracer injections were made into the right sensorimotor cortex. One group of mice $(n=12$; the control group that received vehicle injections beginning on the day of the spinal cord injury) received these injections 30-60 min after the completion of the spinal cord injuries. Another ( $n=7$; the control group implanted with vehicle-containing Alzet minipumps $7 \mathrm{~d}$ after injury) received biotinylated dextran amine (BDA) injections $28 \mathrm{~d}$ after the spinal cord injury. The wild-type littermate controls for Nogo knock-out mice received BDA injections $28 \mathrm{~d}$ after the spinal cord injury.

For the BDA injections, mice were anesthetized as above and placed in a stereotaxic device, the fur was removed by shaving, the scalp was incised, and the skull overlying the sensorimotor cortex was carefully removed with a dental drill. Miniruby BDA [dextran, tetramethylrhodamine, and biotin (mini ruby): molecular weight of 10,$000 ; 10 \%$ in $\mathrm{DH}_{2} \mathrm{O}$ (Invitrogen)] was injected into a total of four sites $(0.4 \mu \mathrm{l}$ per site over a 3-5 min time period) using a $10 \mu$ l Hamilton microsyringe tipped with a pulled glass micropipette. Coordinates were 1.0 lateral, $0.5 \mathrm{~mm}$ deep to the cortical surface, and $+0.5,-0.2,-0.7$, and $1.0 \mathrm{~mm}$ with respect to bregma. After the injections were completed, the skin overlying the skull was sutured with 4-0 silk.

Postoperative care. After the surgeries, the mice were immediately placed on a water-circulating jacketed heating pad. After recovering from the anesthetic, animals were housed four to five per cage on Alpha-Dri bedding. For 10-14 d after surgery, animals received lactated Ringer's solution (5 mg/100 g, s.c.) for hydration, the analgesic Buprnex (Buprenorphine, $0.01 \mathrm{mg} / \mathrm{kg}$ ), and Baytril (Enroflaxacin, $2.5 \mathrm{mg} / \mathrm{kg}$, s.c.) for prophylactic treatment against urinary tract infections (UTIs).

Mice were monitored twice daily for general health, coat quality (indicative of normal grooming activity), and mobility within the cage and for signs of skin lesions on the paralyzed limbs or autophagia of the toes. None of the animals exhibited skin lesions or autophagia throughout the experiment. Bladders of mice with spinal cord injuries were manually expressed twice daily for the duration of the study. Animals were monitored for UTIs for the entire duration of the experiment, and no UTIs were observed. As reported previously, animals were tested behaviorally throughout the postoperative survival period. Testing involved assessment of locomotor function using the Basso-Beattie-Bresnahan scale and the Basso Mouse Scale, inclined grid walking, and kinematic analysis.

Overdose and tissue collection. Mice that received BDA injections at the time of the spinal cord injury were killed 20 or $23 \mathrm{~d}$ after injury. Mice that received BDA injections $28 \mathrm{~d}$ after injury were killed $18 \mathrm{~d}$ later ( $46 \mathrm{~d}$ after the spinal cord injury). Mice were killed by anesthetic overdose and perfused transcardially with PBS, followed by $4 \%$ paraformaldehyde. Spinal cords and brains were removed and immersed in $20 \%$ sucrose for cryoprotection.

Histological assessment. Three tissue blocks were prepared from the spinal cords: (1) a tissue block extending from $\sim 5 \mathrm{~mm}$ above to $5 \mathrm{~mm}$ below the lesion and containing the injury site; (2) the portion of the spinal cord rostral to the tissue block containing the lesion, extending to the spinomedullary junction; and (3) the portion of the spinal cord caudal to the tissue block containing the lesion, extending to caudalmost segment. The main block containing the lesion was sectioned at $20 \mu \mathrm{m}$ in the sagittal plane, collecting every section and maintaining serial order during histological processing. The collection of serial sagittal sections from each animal involved $\sim 70-75$ sections. A few sections from the most lateral part of the spinal cord are typically lost. All sections from the block containing the lesion were stained for BDA as described below. The rostral end of the spinal cord above the injury block and the caudal end below the injury block were sectioned transversely, and 20-30 sections were stained for $\mathrm{BDA}$. The brains were sectioned in the coronal plane, and sections taken every $500 \mu \mathrm{m}$ were stained for BDA.

For BDA staining, sections were washed in PBS and $0.1 \%$ Triton $\mathrm{X}-100$, incubated for $1 \mathrm{~h}$ with avidin and biotinylated HRP (Vectastain $\mathrm{ABC}$ kit; Vector Laboratories), washed in PBS, and then reacted with $\mathrm{DAB}$ in $50 \mathrm{~mm}$ Tris buffer, $\mathrm{pH}$ 7.6, 0.024\% hydrogen peroxide, and $0.5 \%$ nickel chloride.

Cross sections from the rostral block ( $\sim 5 \mathrm{~mm}$ rostral to the lesion) were stained for BDA to determine the extent of CST labeling above the lesion. Cross sections through the caudal block $(\sim 5 \mathrm{~mm}$ caudal to the lesion) were stained for BDA to determine whether any axons in the dCST or dlCST were spared.

To obtain a quantitative measure of the overall extent of CST labeling in each animal, we counted BDA-labeled CST axons in the dCST rostral to the injury. For this purpose, thin (1-3 $\mu \mathrm{m}$ thick) sections were taken $\sim 5 \mathrm{~mm}$ rostral to the injury and mounted on microscope slides before BDA staining. In these thin sections, individual BDA-labeled axons can be counted under high magnification even when packing density is high (as is the case in the main tract). To provide another measure of labeling efficiency, we also counted BDA-labeled axons in the medullary pyramid, through which all CST axons descend. For this purpose, the brainstem was sectioned in the coronal plane, and sections were stained for BDA as above. Counting axons in the medullary pyramid is easier because they are not tightly packed and is advantageous because the pyramid contains the entire CST. In contrast, CST axons in the spinal cord distribute into separate tracts, the dorsal CST in the base of the dorsal column, and the dorsolateral CST (we have not detected BDA-labeled axons in the ventral column in C57BL/6 mice.

\section{Results}

\section{Organization of the CST in uninjured mice}

The organization of the CST in mice as revealed by BDA tracing has been described previously (Steward et al., 2004). After BDA injections into the sensorimotor cortex, the main component of 
BDA-labeled CST axons is in the ventral part of the dorsal column contralateral to the injection (the dorsal CST) (Fig. 1). A second contingent of axons descends in the dorsal portion of the lateral column (the dorsolateral CST). In some mice, a small number of BDA-labeled axons are seen ipsilateral to the injection in the dorsal column and/or the dorsolateral CST representing an uncrossed contingent of CST axons. The number of uncrossed axons varies in individual mice. The pattern of labeling seen in sections rostral to the injury in mice with dorsal hemisection injuries (see figures) is similar to what is seen in uninjured mice.

There was also extensive labeling of terminal arbors in the gray matter on the side of the labeled tracts, and some labeled axons could be seen extending across the midline to the opposite side ipsilateral to the injection forming a recrossing projection. These axons crossed over at different dorsoventral positions in the gray matter. The number of the recrossing axons also varied in individual mice.

With the tracing protocol described here (see Materials and Methods), we did not detect BDA-labeled axons traveling longitudinally in the ventral column ipsilateral to the injection in the position of the ventral CST that is seen in rats. This is consistent with our previous studies in C57BL/6 mice (Steward et al., 2004). BDAlabeled axons can be seen, however, extending down from the main terminal field in the gray matter into the ventral column along fingers of gray matter that contain dendrites of motoneurons (Steward et al., 2004). These axons cannot be followed from section to section, however, as would be possible if the axons extended longitudinally.

It should be noted that, although we have never seen a BDAlabeled ventral CST axon in C57BL/6 mice, we have seen a few ventral CST axons in mice of mixed genetic background. An example is illustrated in supplemental Figure 1 (available at www.jneurosci.org as supplemental material). The one labeled ventral CST axon can be followed from section to section (supplemental Fig. $1 B-D$, available at www.jneurosci.org as supplemental material), indicating that it does travel longitudinally. This axon also has a different morphology than the axons that extend into the ventral column from the labeled arbors in the ventral horn (supplemental Fig. $1 E$, available at www.jneurosci.org as supplemental material). In particular, the ventral CST axon is straight and unbranched and has a generally uniform caliber; the radial axons take a more meandering course, have varicosities, and sometimes can be seen to branch.

Ventral CST axons have also been reported in mice in which the CST is genetically labeled with yellow fluorescent protein (YFP), which are also of mixed genetic background (Bareyre et al., 2005). Analyses of sagittal sections of spinal cords from YFPCST mice confirm that a few YFP-positive axons do extend lon- gitudinally along the ventral column in the position expected for ventral CST axons (B. Zheng, unpublished observations). It may be that these are too few in number to be labeled consistently with BDA or originate from a part of the cortex not included in our BDA injections.

\section{Dorsal hemisections}

Our goal in creating the dorsal hemisection injuries was to transect all components of the CST that are labeled by BDA injections into the sensorimotor cortex, including the dorsal CST in the dorsal column, the dorsolateral CST in the dorsal part of the lateral column, and the axons that travel through the dorsal gray matter, but sparing the ventral white matter. Accordingly, we sought to create lesions that transected the dorsal half of the spinal cord down to a level just below the central canal, extending the lesion laterally at the same level through the dorsal part of the lateral column (Fig. 2A). Approximately $50 \%$ of the mice exhibited lesions that were consistent with our intentions in that the lesions were complete dorsal hemisections and extended just past the central canal. Reconstruction of sagittal sections through the lesion site revealed ballooning of the central canal as is typical 

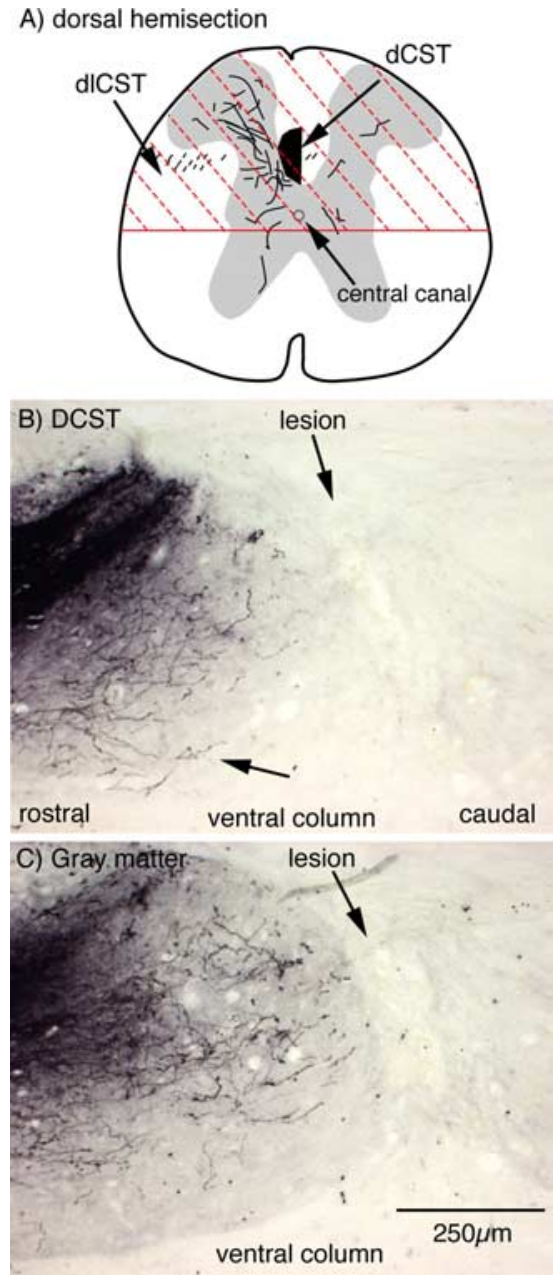

Figure 2. Distribution of CST axons in mice killed $20 \mathrm{~d}$ after a dorsal hemisection: sagittal sections (animal $071405 C$ ). A, Schematic illustration of a dorsal hemisection. B, C, lllustration of two sagittal sections from the block containing the lesion site from the mouse illustrated in Figure 1. B, Sagittal section through the main CST in the dorsal column; $\boldsymbol{C}$, sagittal section through the gray matter lateral to the main tract. The unlabeled arrow in $\boldsymbol{B}$ indicates an axon that extends down into the ventral column. The schematic illustration is modified from Zheng et al. (2006).

when the central canal is damaged. In most animals, most or all of the ventral column and portions of the ventral gray matter were spared. The lesion site was filled with connective tissue (Fig. $2 B, C)$, as is typical for spinal cord lesions in mice, and there was no cavitation in most mice. The lesion site was highly compact, so that the intact parenchyma rostral and caudal to the injury was separated by $<100 \mu \mathrm{m}$ in some mice. In $\sim 15 \%$ of the animals, the lesions were slightly smaller than intended in that the lesion did not extend down to the central canal, although the dorsal column and especially the dCST were completely transected. In the remaining mice, the lesions were somewhat larger than intended, in that there was variable damage to the ventral white matter, and, in one mouse, the lesion was almost a complete transection.

\section{Discerning bona fide from artifactual BDA labeling}

In the course of our studies of the CST after spinal cord injury in mice, we discovered a BDA labeling artifact that can lead to axonal labeling suggestive of long-distance regeneration (Steward et al., 2007). The artifact is seen when tracer injections are made at the same time as a spinal cord injury, which is a common practice to avoid two surgical procedures, and was the procedure used here for animals killed 20-23 d after injury. The labeling occurs as the result of leakage of BDA into the CSF, followed by diffusion to the injury site in the spinal cord in which axons take up the tracer. The most obvious manifestation of the artifact is labeling of large axons in the lateral column, especially on the side ipsilateral to the injection, which has been previously misinterpreted as evidence of robust long-distance regeneration of the CST in Nogo knockout mice (Kim et al., 2003).

As described previously (Steward et al., 2007), the signs indicating artifactual labeling include the following: (1) labeling of axons in ectopic locations rostral and caudal to the lesion, especially in the lateral column; (2) labeled axons that appear hollow and that are less reflective in dark-field illumination than axons labeled by orthograde transport; (3) a higher level of background labeling around the injury site with labeling of axons on both sides of the lesion and labeling of neurons and glia at the injury site; and (4) indications that the injection penetrated the cerebral ventricle, including labeling of ependymal cells lining the ventricle. Evidence of artifactual labeling was seen in 6 of 12 mice that received injections at the time of the spinal cord injury, whereas no artifactual labeling was seen in animals that received BDA injections at $28 \mathrm{~d}$ after injury. In what follows, we consider only those cases that show none of these signs of artifactual labeling.

\section{Dorsal hemisections completely interrupt CST projections to caudal segments}

Figure 1 illustrates an example of the distribution of CST axons in mice killed $20 \mathrm{~d}$ after a dorsal hemisection injury in which the overall extent of orthograde labeling of the CST is robust. The distribution of CST axons in a cross section taken at a high thoracic level rostral to the injury is similar to what is seen in control mice (Fig. 1 $A, C$ ). BDA-labeled axons are prominent in the dorsal CST in the ventral part of the dorsal column contralateral to the injection (the left side of the spinal cord), and a smaller number of labeled axons are present in the dorsolateral CST. There is also a dense plexus of BDA-labeled axons throughout the gray matter on the side of the labeled tract, and some labeled collaterals extend across the midline to the contralateral side (Fig. $1 C$ ). In the mouse illustrated in Figure 1, there are also an unusually large number of BDA-labeled axons in the dorsal and dorsolateral CST ipsilateral to the injection (right side). Although the extent of ipsilateral labeling is unusual, it is useful here because it allows us to document that the dlCST on both sides is transected (Fig. 3).

Despite the fact that the main tracts are heavily labeled in this mouse, longitudinally oriented BDA-labeled axons were not seen in the ventral column in the expected position of the ventral CST (Fig. $1 E$ ). This is in contrast to what is seen in rats, in which ventral CST axons are reliably labeled after BDA injections into the cortex. The few BDA-labeled axons that were present in the ventral column were thin, followed a tortuous trajectory, were beaded, and often could be followed back into the overlying gray matter (Fig. $1 E$ ). In these regards, the axons were quite different in appearance from the axons in the main tracts. Also, most of the BDA-labeled axons in the ventral column were ipsilateral to the main tract (that is, were crossed projections from the contralateral cerebral cortex), whereas a defining criterion of ventral CST axons is that they are uncrossed.

No BDA-labeled axons were seen in a cross section taken below the level of the injury in either the dorsal column, lateral column, or ventral column (Fig. $1 B, D$ ), indicating that all components of the CST that could be detected by BDA labeling were completely transected. Consistent with the absence of artifactual 


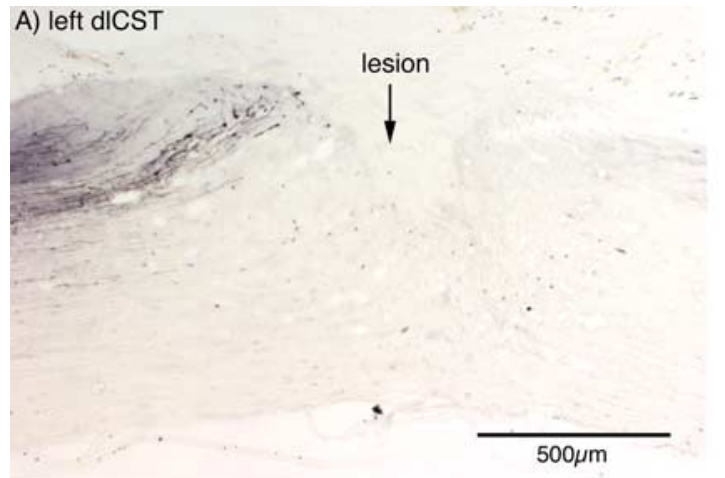

B) right dICST

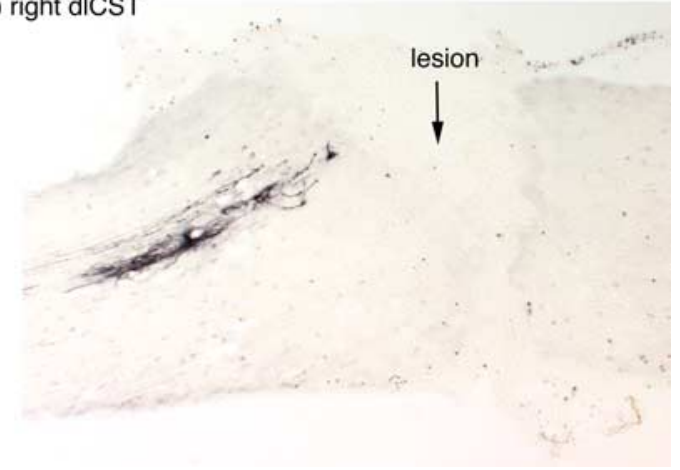

Figure 3. CST axons in the dICST in mice killed $20 \mathrm{~d}$ after a dorsal hemisection (animal 071405C). The panels illustrate sagittal sections through the dICST on each side. $A$, The dICST on the left side. $\boldsymbol{B}$, The dICST on the right side. The BDA injection was made into the right sensorimotor cortex.

labeling in this mouse, there was no indication that the tip of the microsyringe penetrated the cerebral ventricle at the cortical injection site (Fig. 1G).

Sagittal sections of the block containing the lesion site reveal a highly compact lesion extending from the dorsal surface of the spinal cord through the central canal but sparing the ventral columns (Figs. 2-4). Figure $2 A$ illustrates a section through the main CST in the dorsal column; Figure $2 B$ illustrates a section through the gray matter lateral to the main tract. BDA-labeled axons in the main CST in the dorsal column end $\sim 100-200 \mu \mathrm{m}$ from the lesion margin in swollen balls characteristic of axons that have been cut. Similarly, the dense arbors of CST axons in the gray matter also end abruptly at approximately the same distance from the lesion margin (Fig. $2 B$ ), and analysis of serial sections revealed that this was true throughout the mediolateral extent of the spinal cord. A few coarse axons extend from the dense cluster of arbors in the gray matter toward the lesion; many of these end in complex arborized swellings near the lesion margin (Fig. 2 B). BDA-labeled axons in the dorsolateral CST on both sides were also completely transected (Fig. 3) and, like the axons in the main tract, end in swollen balls or complex arborized swellings.

In most mice killed 20-23 d after lesion, all BDA-labeled CST axons ended rostral to the lesion, as described above. The one exception was a mouse killed $23 \mathrm{~d}$ after lesion in which two BDAlabeled axons bypassed the lesion in the ventral column. One of these reentered the gray matter and extended $\sim 400 \mu \mathrm{m}$ caudally (see Fig. 8 below). This appears to be a greatly attenuated version of the pattern of CST axon distribution described below in animals killed at longer postlesion intervals and may indicate axons that are in the process of regenerating, as further described below. The other axon that extended past the lesion ended in the white matter just caudal to the lesion.

\section{Time-dependent extension of CST axons past the lesion via the ventral column and arborization in segments caudal to the injury}

As noted above, we did not observe BDA-labeled axons extending past the lesion through the ventral column in 15 of 16 mice killed 20-23 d after dorsal hemisection injuries. Given this, it was striking that BDA-labeled CST axons were seen extending past the lesion in the ventral column in four of seven of the mice that were killed $46 \mathrm{~d}$ after injury (see Figs. 4-6). The trajectory of these BDA-labeled axons was highly unusual and suggested that they had grown from rostral segments past the injury site into caudal regions.

An example of CST axons bypassing the lesion via the ventral column is illustrated in Figure 4. The lesion in this mouse was a complete hemisection; labeled axons in the dorsal CST and dorsolateral CST were completely transected. Nevertheless, several BDA-labeled axons could be seen bypassing the lesion site in the ventral column (Fig. 4, arrows). The labeled axons in the ventral column could be traced back into the gray matter rostral to the injury in which they intermingled with the dense plexus of labeled CST axons proximal to the lesion (Fig. $4 A, B$ ) and could be followed caudally past the lesion in which they reentered the gray matter of caudal segments (Fig. $4 E, F$ ). Figure $4 G$ illustrates a reconstruction of the BDA-labeled axons through serial sections, which revealed complex arbors in the gray matter caudal to the injury. Although this drawing illustrates the distribution of BDAlabeled axons, it does not fully capture the full complexity of the individual axon arbors.

In the mouse illustrated in Figure 4, two BDA-labeled axons extended to the caudal end of the sagittal section in which they formed complex arbors, and branches from these arbors extended to the cut edge of the section (Fig. $5 E$, arrow). The measured distance from the lesion to the caudal end of the sagittal section was $4 \mathrm{~mm}$ (Table 1 ), but this does not take into account any shrinkage of the section that would have occurred during processing. The extensions of these axons could be seen in the cross sections taken $\sim 5 \mathrm{~mm}$ caudal to the injury (Fig. $5 F$ ). In the other mice in which BDA-labeled axons were seen bypassing the lesion, the arbors in caudal segments were concentrated within 1-2 $\mathrm{mm}$ of the lesion, but a few axons extended farther (Table 1 shows the maximal caudal extension seen in the different mice).

Several lines of evidence strongly suggest that the axons that bypassed the lesion via the ventral column represent regenerated CST axons rather than ventral CST axons. First, BDA-labeled axons were not seen extending longitudinally along the ventral column in cross sections taken $\sim 5 \mathrm{~mm}$ rostral to the injury (Fig. $5 A, C)$. Second, the BDA-labeled axons that were present in the ventral column rostral to the injury were ipsilateral to the main tract (Fig. 5C) and thus were crossed axons, not uncrossed axons as would be expected for the ventral CST. Third, the axons entered the ventral column from the gray matter rostral to the injury. The axons could not be traced through several consecutive serial cross sections as would be possible if the axons were traveling longitudinally along the ventral column. Finally, BDAlabeled axons were not seen extending longitudinally along the ventral column in cross sections taken $\sim 5 \mathrm{~mm}$ caudal to the injury (Fig. $5 B, D$ ), indicating that the axons seen near the lesion site in sagittal sections were not spared ventral CST axons (spared axons would be expected to extend longitudinally into caudal segments). Other ongoing studies of YFP-CST mice confirm that, when YFP-labeled axons are seen in the ventral column, they extend longitudinally and do not take the trajectories of the un- 


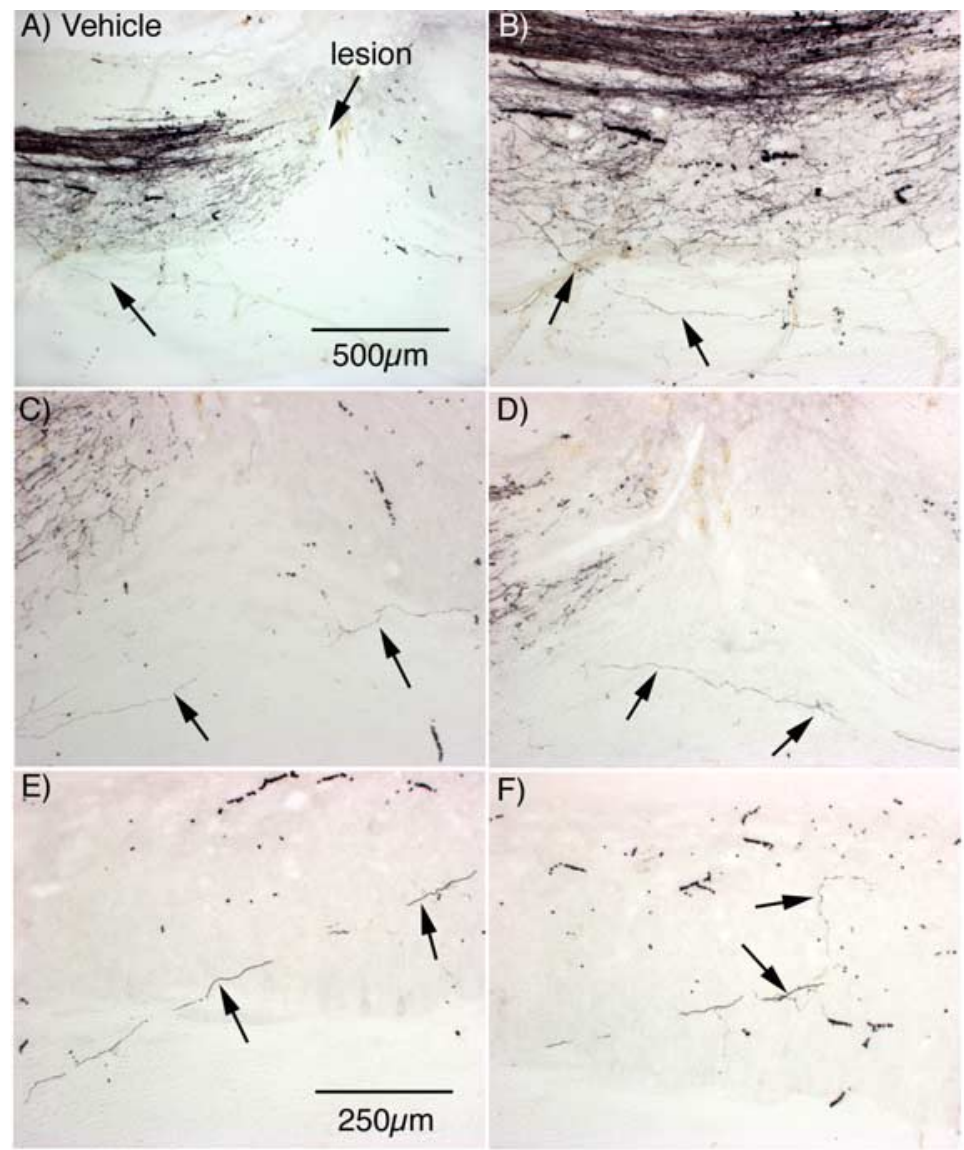

G) Reconstruction of axons that pass the lesion

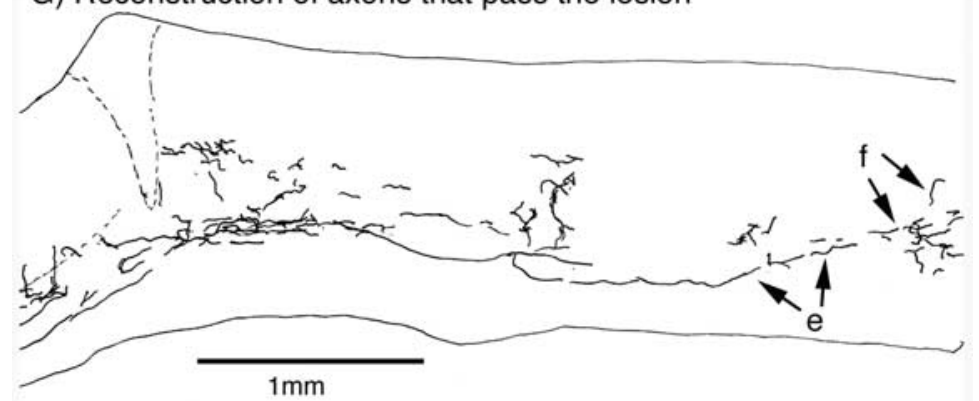

Figure 4. Extension of CST axons past the lesion via the ventral column in mice killed $46 \mathrm{~d}$ after a dorsal hemisection: sagittal sections (animal 060106l). $A$, Sagittal section through the dCST in the dorsal column. Note axon extending down into the ventral column (arrow). $\boldsymbol{B}$, Higher-magnification view of the section shown in $\boldsymbol{A}$. Arrows indicate the axon extending from the gray matter into the ventral column. $\boldsymbol{C}, \boldsymbol{D}$, High-magnification views of BDA-labeled axons in the ventral column just below the lesion site. $\boldsymbol{E}$, Axon reentering the gray matter from the ventral column caudal to the lesion. $\boldsymbol{F}$, Complex terminal arbor in the gray matter. $\boldsymbol{G}$, Drawing of labeled axons from serial sagittal sections. e and findicate the labeled axons shown in $\boldsymbol{E}$ and $\boldsymbol{F}$. lesion into caudal segments (Fig. 7B). The composite image was created by piecing together segments of the axon from four serial sections. Because of the density of the labeled axon arbors in the gray matter, we were unable to determine definitively whether the axon originated from the dCST or as a branch from one of the arbors in the gray matter. Definitive determination of the origin of these axons will require the use of tracing techniques that label a smaller number of axons so that the axons do not become lost in the thicket of labeled arbors in the gray matter.

In all four of the seven mice killed $46 \mathrm{~d}$ after lesion, most of the BDA-labeled axons that bypassed the lesion did so via the medial portion of the ventral column very near the midline, and all were ipsilateral to the main tract. In one mouse, one of the axons bypassed the lesion via the lateral portion of the ventral column. Similar examples of axons bypassing a dorsal hemisection lesion were seen in mice that were the homozygous wild-type controls for mice lacking Nogo that received BDA injections $28 \mathrm{~d}$ after injury (data not shown).

Although striking in appearance, the overall number of axons that bypassed the lesion via the ventral column was small. Nine axons were seen bypassing the lesion in the case illustrated in Figures 4 and 5, and seven were seen in the case illustrated in Figures 6 and 7. To put this in perspective, counts of BDA-labeled axons rostral to the injury in the mouse illustrated in Figures 4 and 5 revealed a total of 1335 labeled axons in the medullary pyramid (see Table 1). The mouse illustrated in Figures 6 and 7 had a total of 482 labeled axons in the medullary pyramid. The two other mice had seven and two BDAlabeled axons that bypassed the lesion via the ventral column (mean of 6.3 per mouse) and 524 and 377 BDA-labeled axons in the medullary pyramid, respectively. Thus, the axons that bypassed the lesion via the ventral column represent usual axons seen here (J. K. Lee, S. Y. Chow, A. F. Chan, and B. Zheng, unpublished observations). Together, these observations argue strongly that the axons that bypassed the lesion site via the ventral column were not spared axons of the ventral CST.

The axons that bypassed the lesion generally extended ventrally from the field of axon arbors in the gray matter rostral to the injury (Fig. 4B). Because this trajectory is strikingly different from what would be expected if these were ventral CST axons, additional examples from a different case are illustrated in Figures 6 and 7. The images in Figure 6 show three adjacent sections near the midline and illustrate a region $\sim 1-3 \mathrm{~mm}$ from the lesion site. Figure 7 illustrates a reconstruction of a single axon that emerges from the gray matter $\sim 1 \mathrm{~mm}$ rostral to the injury into the ventral column (Fig. $7 A$ ) and then extends ventral to the only $\sim 1 \%$ of the total number of BDA-labeled axons in the medullary pyramid.

In three of seven mice, no BDA-labeled axons were seen to extend past the lesion. One possible explanation is that the overall level of CST labeling was lower in these mice than in the mice in which regenerating axons were seen. As another quantitative measure of overall labeling efficiency, we counted the number of BDA-labeled axons in the dCST in cross sections taken $\sim 5 \mathrm{~mm}$ rostral to the injury (Table 1 ). The four mice with regenerated CST axons had 1753 (the animal illustrated in Figs. 4, 5), 1106, 910, and 467 BDA-labeled axons in the dCST, whereas the three mice in which no BDA-labeled axons extended past the lesion had 82, 522, and 1248 labeled axons in the dCST. Because the overall level of CST labeling was lower in at least one of the mice 
without regenerated axons, it is possible that the overall incidence of CST regeneration may be higher than our sample suggests.

\section{Evidence of early regenerative growth at 20-23 d after injury}

The trajectory of the axons that bypassed the lesion via the ventral column in animals killed $46 \mathrm{~d}$ after injury was highly suggestive of regeneration. If these are regenerated axons, however, there should be evidence of axons in intermediate locations (that is, in the process of regenerating) at earlier postlesion intervals. Accordingly, we went back to the cases killed at 20-23 d after lesion to search for evidence of axons that might be in the process of regenerating.

Sagittal sections from near the midline from the animal illustrated in Figures 2 and 3 revealed a few BDA-labeled axons that extended down from the gray matter rostral to the injury into the ventral column (Fig. 8). Two serial sections are shown through the main dCST. In both sections, BDA-labeled axons can be seen extending into the ventral column from the gray matter rostral to the injury (arrows). These labeled axons are consistent with what would be expected if axons were in the process of regenerating down into the ventral column on their way to caudal segments. None of these axons extended past the lesion, however.

Figure 9 illustrates a composite reconstruction of a single axon that bypassed the lesion from a different mouse killed at $23 \mathrm{~d}$ after lesion in which BDA labeling was robust and in which the lesion completely transected all BDA-labeled axons in the dCST and dlCST, as evidenced by a complete absence of BDA-labeled axons in cross sections taken $\sim 5 \mathrm{~mm}$ caudal to the injury (data not shown). As in Figure 7, the composite reconstruction was created by piecing together images of fragments of the axon seen in serial sections. The reconstructed axon (axon 1) extends from the gray matter into the ventral column in which it ends just ventral to the lesion in a slight swelling that could represent a growth cone. The axon could be traced back to a location very near the cut axons in the dCST, but we were unable to determine definitively whether the axon originated from the dCST or as a branch from one of the arbors in the gray matter. Other examples of BDA-labeled axons extending from the ventral gray matter down into the ventral column were seen in other sections (data not shown). All axons that extended into the ventral column were thin, varicose, and followed a meandering course, and some branched near their ends (like the axon shown in Fig. $8 \mathrm{~B}$ ). Most importantly, all of the axons were located in the ventral column ipsilateral to the main labeled tract. In all of these respects, the axons in the ventral column had a different morphology than would be expected if they were spared axons of the ventral CST. This morphology is, however, consistent with the interpretation that axons sprout down into the ventral column and are in the process of extending farther caudally.

The mouse illustrated in Figure 9 was the only one killed 20-23 d after lesion in which any BDA-labeled axons were seen to bypass the lesion. One of the two axons that bypassed the lesion is axon 2 in Figure 9. This axon could be traced through nine serial sections back to the point at which it bypassed the lesion via the ventral column near the midline. It extended $\sim 600 \mu \mathrm{m}$ caudal to the lesion. The photographic reconstructions of axon 2 were not satisfactory because, throughout most of its course, the axon was very thin and tortuous.

\section{Discussion}

The present study provides compelling evidence for a form of naturally occurring regenerative growth of CST axons after spinal cord injury that has not been described previously (growth around the lesion via the ventral column). This form of regenerative growth was seen in the majority of mice killed $46 \mathrm{~d}$ after injury but not in mice killed 20-23 d after injury.

The only alternative explanation for the findings is that the axons we claim to be regenerated actually exist in normal mice and are spared by the lesion. The possibility that these are spared axons is very unlikely for several reasons. First, the axons that 
Table 1. Quantification of BDA-labeled CST axons

\begin{tabular}{|c|c|c|c|c|c|}
\hline Animal & Survival & $\begin{array}{l}\text { Axons in } \\
\text { pyramid }\end{array}$ & $\begin{array}{l}\text { Axons } \\
\text { in } \\
\text { dCST }\end{array}$ & $\begin{array}{l}\text { Axons bypassing the lesion in } \\
\text { the ventral column }\end{array}$ & $\begin{array}{l}\text { Maximal distance } \\
\text { of growth }\end{array}$ \\
\hline $071405 C$ & $20 d$ & 847 & 1246 & 0 & 0 \\
\hline 071405E & $20 d$ & 544 & 1114 & 0 & 0 \\
\hline 0714051 & $20 d$ & 306 & 1168 & 0 & 0 \\
\hline 071405M & $20 d$ & 84 & 911 & 0 & 0 \\
\hline 0714050 & $20 d$ & 35 & 444 & 0 & 0 \\
\hline 071405R & $20 d$ & 24 & 524 & 0 & 0 \\
\hline 071405V & $20 \mathrm{~d}$ & 688 & 1542 & 0 & 0 \\
\hline 071405Y & $20 d$ & 130 & 1318 & $\begin{array}{l}0 \\
0 \% \text {; mean of } 0\end{array}$ & 0 \\
\hline $121405 B$ & $23 d$ & 38 & 289 & 0 & 0 \\
\hline $121405 D$ & $23 d$ & 659 & 1171 & 0 & 0 \\
\hline $121405 E$ & $23 d$ & 47 & 288 & 0 & 0 \\
\hline $121405 G$ & $23 d$ & 441 & 1258 & 0 & 0 \\
\hline $121405 \mathrm{I}$ & $23 d$ & 162 & 504 & 0 & 0 \\
\hline $121405 \mathrm{~L}$ & $23 d$ & 526 & 956 & 2 & $0.6 \mathrm{~mm}$ \\
\hline $121405 \mathrm{M}$ & $23 d$ & 1776 & 974 & 0 & 0 \\
\hline 1214050 & $23 d$ & 476 & 1562 & $\begin{array}{l}0 \\
1 \text { of } 8(12.5 \%) ; \text { mean of } 0.25\end{array}$ & 0 \\
\hline 010606A & $46 d$ & 172 & 82 & 0 & 0 \\
\hline $010606 \mathrm{~F}$ & $46 \mathrm{~d}$ & 361 & 522 & 0 & 0 \\
\hline 0106061 & $46 d$ & 1335 & 1106 & 9 & $4 \mathrm{~mm}+$ \\
\hline 010606L & $46 d$ & 482 & 910 & 7 & $2.3 \mathrm{~mm}$ \\
\hline 0106060 & $46 d$ & 377 & 467 & 2 & $1.8 \mathrm{~mm}$ \\
\hline 010606R & $46 d$ & 524 & 1753 & 7 & $4 \mathrm{~mm}+$ \\
\hline 010606V & $46 d$ & 557 & 1248 & $\begin{array}{l}0 \\
4 \text { of } 7(57 \%) ; \text { mean of } 3.54\end{array}$ & $\begin{array}{l}0 \\
0\end{array}$ \\
\hline
\end{tabular}

extend past the lesion do not have a trajectory that would be consistent with their being spared axons of the ventral CST, which is the only known component of corticospinal axons in the ventral column. Rather than extending longitudinally along the ventral column, the axons extend from the gray matter rostral to the injury, project in a curvilinear manner caudally into the ventral column bypassing the lesion ventrally, and then reenter the gray matter in caudal segments in which they form elaborate terminal arbors. Importantly, the axons that bypass the lesion in the ventral column are ipsilateral to the main tract, whereas ventral CST axons should be contralateral to the main tract and ipsilateral to the cortex of origin as in rats and other species. Finally, in animals killed 20-23 d after lesion, there were profiles suggestive of axons in the process of growing, but there was essentially no extension beyond the lesion in contrast to what was at $46 \mathrm{~d}$ after injury. Thus, the CST axons that bypass the lesion via the ventral column fulfill most of the criteria for regenerated axons (Steward et al., 2003).

The only possible alternative explanation for our findings is that a corticospinal pathway exists in mice that has not been seen in any other species, that the axons in this pathway do not transport BDA in uninjured mice, and that they become capable of BDA transport after a spinal cord injury. This explanation postulates a corticospinal pathway that has never been demonstrated, a change in BDA transport capabilities that occurs through unknown mechanisms, and still does not account for the fact that the axons are not evident at 20-23 d after injury and are dramatically evident at $46 \mathrm{~d}$. Given the facts, this alternative explanation seems far more remote than the possibility that these are actually regenerated axons. Together, our results make a strong case that
CST axons are capable of growing several millimeters and forming elaborate terminal arbors after spinal cord injury in mice.

\section{Regeneration, regenerative sprouting, or sprouting?}

We use the term "axon regeneration" to refer to a situation in which an axon forms a growth cone at the cut end and grows out and "regenerative sprouting" to refer to a situation in which growing axons originate as collaterals from a cut axon (Steward, 2006). Sprouting refers to a situation in which growing axons originate as collaterals or from terminal arbors that may or may not arise from axons that were cut. The key to distinguishing between these forms of regenerative growth is determining whether the growing axons originate from axons that were cut. In the present experiments, it was not possible to trace the axons in the ventral column back to their point of origin because they became lost in the dense terminal arbors in the gray matter rostral to the injury. Thus, it is not possible to say with certainty whether the axons arose from an axon that had been cut or as collaterals from the terminal arbors of CST axons in the gray matter. In any case, the axons extended for several millimeters caudal to the injury, which represents regenerative growth over several spinal segments.

The growing axons extend along surviving white matter bridges that remain intact after a partial spinal cord injury. Thus, molecules present in intact myelin do not represent an insurmountable barrier to regenerating CST axons. This is consistent with previous studies indicating that axons from young neurons transplanted into the CNS are capable of extending for long distances through normal white matter tracts (Davies et al., 1999). Conversely, profiles that have the characteristics of growing ax- 

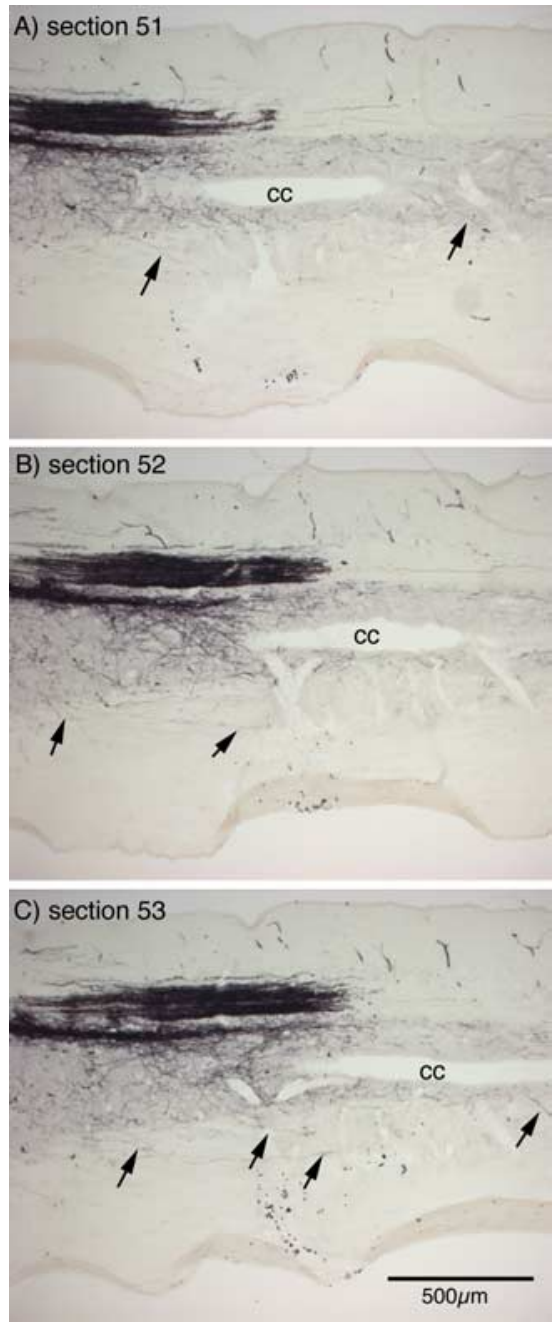

Figure 6. CST axons that extend past the lesion via the ventral column originate as collaterals from the gray matter in rostral segments (animal 060106L). $\boldsymbol{A}-\boldsymbol{C}$ illustrate three adjacent sagittal sections taken near the midline (identified as the center of the central canal). The panels illustrate a region $\sim 1-3 \mathrm{~mm}$ rostral to the lesion (which is just to the right out of the frame of the photomicrograph). Arrows indicate axons that extend ventrally into the ventral column from the gray matter. cc, Central canal.

ons (that is, with complex endings suggestive of frustrated growth cones) invariably avoid the area of the lesion itself. These observations confirm previous findings that molecules in the lesion site present a more formidable barrier to regenerating axons than molecules present in intact myelin (Davies et al., 1999).

It is of interest to speculate why the axons seen here are concentrated in the ventral column near the midline. This is where axons of the ventral CST would travel, although on the opposite side. In this regard, it is noteworthy that CST axons in all species actually travel in different tracts. For example, in rats, CST axons descend in the dCST, the dICST, and the ventral CST. It may be that specificity cues exist providing a hierarchy of preferences so that, when growing axons are prevented from extending along one pathway, they take the next preferred route. This would predict that, if the dorsal part of the lateral column was spared, in which dlCST axons are found, growing CST axons might take this route after a dorsal column injury. This is in fact seen (more on this below). In our experiments, the dorsal part of the lateral column is transected, however, leaving the medial part of the ventral column in which ventral CST axons would travel. Al-

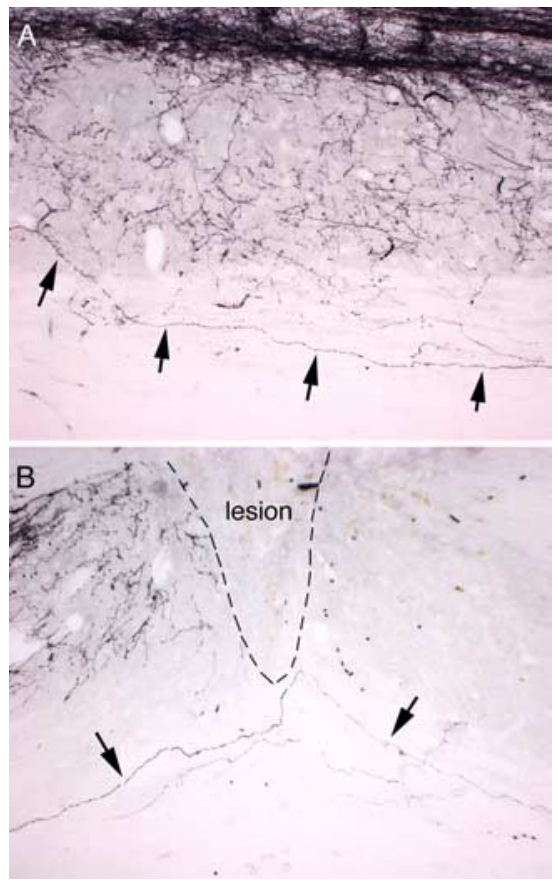

Figure 7. Composite reconstruction of CST axons extending past the lesion via the ventral column (animal 060106L). The panels illustrate composite reconstructions of an axon that emerges from the ventral gray matter $\sim 1 \mathrm{~mm}$ rostral to the injury ( $A$, arrows) and extends along the ventral column bypassing the lesion $(\boldsymbol{B})$. The images were constructed by taking images of segments of the axon from four adjacent sections and superimposing them on a single image. A second, smaller axon is partially reconstructed in $\boldsymbol{B}$ (below the main axon indicated by arrows). Note the irregular diameter and tortuous course of the axons as they pass the lesion.

though speculative, this explains our data and also why other studies have reported CST growth along the dorsal part of the lateral column.

\section{Comparison with forms of naturally occurring CST axon growth reported in previous studies}

The exact form of regenerative growth that we describe here has not to our knowledge been reported previously in the absence of some therapeutic or molecular intervention (more on this below). The growth of the CST that we describe here has three components: (1) regeneration or sprouting in rostral segments; (2) extension ventrally into the ventral column and then continuing past the lesion; and (3) formation of terminal arbors in caudal segments. Although this growth response has not been described previously in its entirety, the first and third components of the response have been reported.

Sprouting of CST axons rostral to a spinal cord injury has been reported previously in rats (Fouad et al., 2001), although the exact nature of the growth response may be different. Fouad et al. reported sprouting of CST axons in cervical segments after thoracic level injuries, whereas here, the formation of new CST axons occurs primarily in segments just rostral to the injury. We did not assess CST axons in cervical segments, and so the form of growth that Fouad et al. reported may also have occurred here. CST sprouting in rostral segments has also been described after dorsal hemisection lesions in SJL/J mice (Sicotte et al., 2003), but extension of sprouts into the ventral column was not described. This form of CST sprouting rostral to an injury is involved in the formation of the intraspinal relay via propriospinal neurons that occurs spontaneously in rats (Bareyre et al., 2004). 

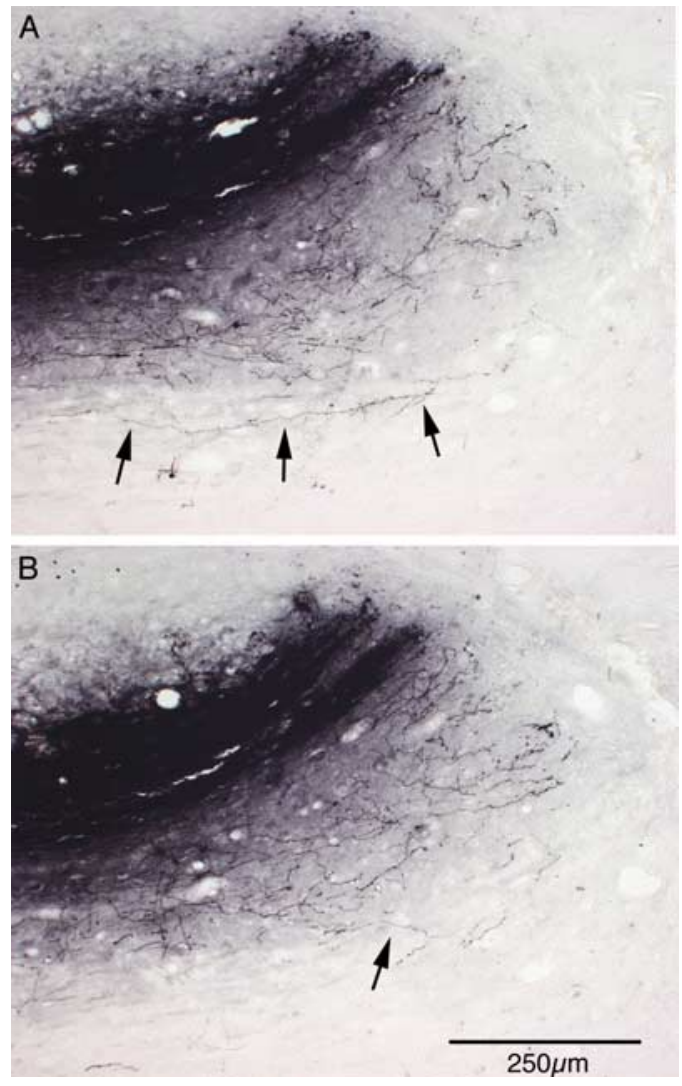

Figure 8. Axons that extend into the ventral column in a mouse killed $20 \mathrm{~d}$ after a dorsal hemisection: sagittal sections (animal 071405C). The panels illustrate two adjacent sagittal sections taken through the main labeled tract in the dorsal column. These are from the same mouse illustrated in Figure 1. Arrows indicate axons that extend from the gray matter into the ventral column.

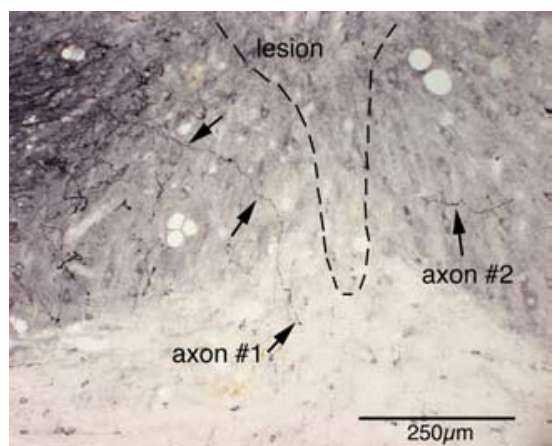

Figure 9. Axons that extend into the ventral column in a mouse killed $23 \mathrm{~d}$ after a dorsal hemisection: sagittal sections (animal 121405L). The panel illustrates a composite reconstruction of an axon (axon \#1) that extends from a position very near the cut axons in the dCST and extends ventrally into the ventral column. The images were constructed by taking images of segments of the axon from four adjacent sections and superimposing them on a single image. This axon ended in the small swelling indicated by the arrow and label (axon\#1). A segment of another axon can be seen in the gray matter caudal to the lesion (axon \#2), the course of which is described in Results.

A circuit similar to what we describe here has been described as a result of sprouting of ventral CST axons after dorsal lesions that spare the ventral column. A ventral CST is reliably seen in rats (Brösamleand Schwab, 2000), and dorsal lesions spare these axons. After dorsal lesions at the cervical level, spared axons of the ventral CST sprout in segments caudal to the injury increasing the net CST input to neurons in caudal segments (Weidner et al.,
2001). The sprouting response is different from what is shown here because there is no evidence that the response involves growth of axons past the lesion. Also, the circuit formed as a result of sprouting of normal ventral CST axons differs from what is described here, in that ventral CST axons are ipsilateral to the cortex of origin whereas the axons shown here are contralateral.

\section{Comparison with CST axon growth after genetic manipulations or therapeutic interventions}

Three studies have assessed CST axon regeneration after dorsal hemisection injuries in Nogo knock-out versus wild-type mice. In one study (Zheng et al., 2003), there was no significant enhancement of CST axon growth in mice lacking Nogo, whereas two other studies reported enhanced CST axon growth in different lines of Nogo knock-out mice. Kim et al. (2003) focused primarily on BDA-labeled axons in ectopic locations in the lateral column. We have shown that similar patterns of labeling can be attributable to an artifact (Steward et al., 2007), although a follow-up report describes other evidence of enhanced CST axon growth in Nogo knock-out mice (Cafferty et al., 2007). In the third study (Simonen et al., 2003), the lesions were slightly smaller and spared the dorsal part of the lateral column (and thus the dlCST). The axons that extended past the lesion in these Nogo-A knock-out mice projected via the dorsal part of the lateral column and dorsolateral gray matter, and thus followed the route taken by axons traveling via the dlCST. The extent of this form of regenerative growth varies when the Nogo-A mutant allele is present in different strains of mice (Dimou et al., 2006). Importantly, the form of growth shown here involving extension along the ventral column differs from what is reported in any of the previous studies involving Nogo knock-out mice. An important implication from our current study is that, with a small number of axons passing around the lesion, one observes what could be interpreted as a major effect on axon regeneration (Fig. $5 G)$. Therefore, even a small degree of sparing of CST axons including those of the dlCST may account for major differences in the appearance of axon regeneration between individual mice in any particular experiment. Such variables need to be carefully considered in interpreting experimental results involving a lesion that incompletely severs labeled CST axons.

The growth reported here superficially resembles the regenerative growth of CST axons that has been reported after treatment with the "IN-1 antibody," which recognizes Nogo and other proteins. Brösamle et al. (2000) report extension of CST axons past a T9 dorsal hemisection lesion in 11 of 18 rats treated with a humanized IN-1 antibody, which was not seen in 12 untreated control rats. The axons bypassed the lesion ventrally, although the drawings in the paper suggest that they take a more dorsal route than spared ventral CST axons. The BDA injections into the cortex were bilateral in the experiments by Brösamle et al., and so it was impossible to determine whether the axons that bypass the lesion were ipsilateral or contralateral to the cortex of origin.

Studies using a more specific antibody against Nogo report increases in the number of axons that project past the lesion via the dorsolateral gray matter (Liebscher et al., 2005). In this study, a T-shaped lesion was made to completely sever CST axons in the dorsal column (dCST), dorsal part of the lateral column (dlCST), and ventral column (site of the ventral CST in rats). Some CST axons normally extend caudally via the dorsolateral gray matter, and thus the regenerative growth appears to involve an increase in axons taking this normal trajectory.

Axons with the trajectory described here were seen also described in mice treated with a Nogo receptor antagonist peptide 
(NEP1-40) but not in vehicle-treated control mice (Li and Strittmatter, 2003). In our repeat experiment, however, mice with this trajectory were seen in some mice in all treatment groups (Steward et al., 2008). Thus, either the reported increase (Li and Strittmatter, 2003) was not attributable specifically to treatment with NEP1-40, or, if it was, it may be an acceleration of a growth response that can occur normally rather than the induction of growth that would otherwise be impossible.

The distribution of regenerated CST axons shown here also resembles the distribution of some of the axons seen in rats treated with Ibuprofen after dorsal hemisection injuries at T6-T7 (Fu et al., 2007). Their reconstructions of sagittal sections indicate that labeled CST axons bypassed the lesion via the ventral column, taking trajectories that are similar to the ones shown here. Fu et al. traced CST axons by making bilateral BDA injections into the cortex, and so it is impossible to determine whether the axons that bypassed the lesion via the ventral column were from the ipsilateral or contralateral cerebral cortex. This is a critical issue because, if the axons are ipsilateral, they are in the same location as spared ventral CST axons. This is a different situation than what we report here, namely axons in the ventral column that are ipsilateral to the main labeled tract (and thus contralateral to the cortex of origin) in a location in which CST axons are not seen in uninjured animals. The former situation always leaves open the possibility of enhanced axon sparing or enhanced axonal transport as a result of the therapeutic intervention. This possibility is essentially eliminated when axons are seen with entirely novel trajectories.

Interestingly, one study (Sicotte et al., 2003) reports the existence of a ventral CST in SJL/J mice as revealed by tract tracing using wheat germ agglutinin (WGA)-HRP and provides evidence for sprouting of ventral CST axons in caudal segments after immunization with myelin proteins (Nogo-66 and MAG). The images of ventral CST axons shown in Sicotte et al. are different than anything we have seen in C57BL/6 mice, which may indicate a strain difference in CST organization. Alternatively, the difference could be attributable to the injection parameters or the tract tracing method (WGA-HRP in the Sicotte et al. study vs BDA here).

\section{Implications for studies in mice involving therapeutic interventions and genetic modifications}

The naturally occurring CST regeneration after spinal cord injury that we document here is of major significance for studies in mice that involve therapeutic interventions or genetic modifications designed to enhance regeneration. Although the regenerated axons represent only a small percentage of the total number of labeled CST axons, they are quite striking in appearance and form elaborate terminal arbors in segments caudal to the injury. Consideration must now be given to the possibility that putative regeneration that is seen as a consequence of a genetic modification or therapeutic intervention is an enhancement or acceleration of a naturally occurring response rather than an induction of growth that is beyond the realm of possibility in normal mice. In addition, variations in this form of spontaneous regeneration among individual mice in a particular experiment must be considered in interpreting data that involve a limited number of animals. It remains to be seen whether this growth capability is unique to mice. If so, translating treatments that target particular molecular mechanisms to enhance axon regeneration from mice to other species including man may be more challenging than previously appreciated. Conversely, mice may have a unique ad- vantage of providing a sensitized background in which the effect of any regeneration enhancing treatment can be more readily assessed.

\section{References}

Bareyre FM, Kerschensteiner M, Raineteau O, Mettenleiter TC, Weinmann O, Schwab ME (2004) The injured spinal cord spontaneously forms a new intraspinal circuit in adult rats. Nat Neurosci 7:269-277.

Bareyre FM, Kerschensteiner M, Misgeld T, Sanes JR (2005) Transgenic labeling of the corticospinal tract for monitoring axonal responses to spinal cord injury. Nat Med 11:1355-1360.

Brösamle C, Schwab ME (2000) Ipsilateral, ventral corticospinal tract of the adult rat: ultrastructure, myelination and synaptic connections. J Neurocytol 29:499-507.

Brösamle C, Huber AB, Fiedler M, Skerra A, Schwab ME (2000) Regeneration of lesioned corticospinal tract fibers in the adult rat induced by a recombinant, humanized IN-1 antibody fragment. J Neurosci 20:8061-8068.

Bulsara KR, Iskandar BJ, Villavicencio AT, Skene JH (2002) A new millenium for spinal cord regeneration: growth-associated genes. Spine 27:1946-1949

Cafferty WB, Kim JE, Lee JK, Strittmatter SM (2007) Response to correspondence: Kim et al., "axon regeneration in young adult mice lacking Nogo-A/B,” Neuron 38, 187-199. Neuron 54:195-199.

Davies SJ, Goucher DR, Doller C, Silver J (1999) Robust regeneration of adult sensory axons in degenerating white matter of the adult rat spinal cord. J Neurosci 19:5810-5822.

Dimou L, Schnell L, Montani L, Duncan C, Simonen M, Schneider R, Liebscher T, Gullo M, Schwab ME (2006) Nogo-A-deficient mice reveal strain-dependent differences in axonal regeneration. J Neurosci 26:5591-5603.

Fernandes KJ, Fan DP, Tsui BJ, Cassar SL, TetzlaffW (1999) Influence of the axotomy to cell body distance in rat rubrospinal and spinal motoneurons: differential regulation of GAP-43, tubulins, and neurofilament-M. J Comp Neurol 414:495-510.

Fitch MT, Silver J (2008) CNS injury, glial scars, and inflammation: Inhibitory extracellular matrices and regeneration failure. Exp Neurol 209:294-301.

Fouad K, Pedersen V, Schwab ME, Brösamle C (2001) Cervical sprouting of corticospinal fibers after thoracic spinal cord injury accompanies shifts in evoked motor responses. Curr Biol 11:1766-1770.

Fu Q, Hue J, Li S (2007) Nonsteroidal anti-inflammatory drugs promote axon regeneration via RhoA inhibition. J Neurosci 27:4154-4164.

Hains BC, Black JA, Waxman SG (2003) Primary cortical motor neurons undergo apoptosis after axotomizing spinal cord injury. J Comp Neurol 462:328-341.

Harel NY, Strittmatter SM (2006) Can regenerating axons recapitulate developmental guidance during recovery from spinal cord injury? Nat Rev Neurosci 7:603-616.

He Z, Koprivica V (2004) The Nogo signaling pathway for regeneration block. Annu Rev Neurosci 27:341-368.

Kim JE, Li S, GrandPré T, Qiu D, Strittmatter SM (2003) Axon regeneration in young adult mice lacking Nogo-A/B. Neuron 38:187-199.

Li S, Strittmatter SM (2003) Delayed systemic Nogo-66 receptor antagonist promotes recovery from spinal cord injury. J Neurosci 23:4219-4227.

Liebscher T, Schnell L, Schnell D, Scholl J, Schneider R, Gullo M, Fouad K, Mir A, Rausch M, Kindler D, Hamers FP, Schwab ME (2005) Nogo-A antibody improves regeneration and locomotion of spinal cord-injured rats. Ann Neurol 58:706-719.

McDonald JW (1999) Repairing the damaged spinal cord. Sci Am 281:64-73.

Schwab JM, Brechtel K, Mueller CA, Failli V, Kaps HP, Tuli SK, Schluesener HJ (2006) Experimental strategies to promote spinal cord regeneration-an integrative perspective. Prog Neurobiol 78:91-116.

Sicotte M, Tsatas O, Jeong SY, Cai CQ, He Z, David S (2003) Immunization with myelin or recombinant Nogo-66/Mag in alum promotes axon regeneration and sprouting after corticospinal tract lesion in the spinal cord. Mol Cell Neurosci 23:251-263.

Silver J, Miller JH (2004) Regeneration beyond the glial scar. Nat Rev Neurosci 5:146-156.

Simonen M, Pedersen V, Weinmann O, Schnell L, Buss A, Ledermann B, Christ F, Sansig G, van der Putten H, Schwab ME (2003) Systemic dele- 
tion of the myelin-associated outgrowth inhibitor Nogo-A improves regenerative and plastic responses after spinal cord injury. Neuron 38:201-211.

Steward O (2006) Anatomical and biochemical plasticity of neurons: regenerative growth of axons, sprouting, pruning, and denervation supersensitivity. In: Textbook of neural repair and rehabilitation, Vol I, Neural repair and plasticity (Selzer M, Clarke S, Cohen L, Duncan P, Gage F, eds), pp 5-25. New York: Cambridge UP.

Steward O, Zheng B, Tessier-Lavigne M (2003) False resurrections: distinguishing regenerated from spared axons in the injured CNS. J Comp Neurol 459:1-8.

Steward O, Zheng B, Ho C, Anderson K, Tessier-Lavigne M (2004) The dorsolateral cortico-spinal tract in mice: an alternative route for corticospinal input to caudal segments following dorsal column lesions. J Comp Neurol 472:463-477.

Steward O, Zheng B, Banos K, Yee KM (2007) Response to: Kim et al., "axon regeneration in young adult mice lacking Nogo-A/B," Neuron 38, 187199. Neuron 54:191-195.

Steward O, Sharp K, Yee KM, Hofstadter M (2008) A re-assessment of the effects of a Nogo-66 receptor antagonist on regenerative growth of axons and locomotor recovery after spinal cord injury in mice. Exp Neurol 209:446-468.

Weidner N, Ner A, Salimi N, Tuszynski MH (2001) Spontaneous corticospinal axonal plasticity and functional recovery after adult central nervous system injury. Proc Natl Acad Sci U S A 98:3513-3518.

Yiu G, He Z (2006) Glial inhibition of CNS axon regeneration. Nat Rev Neurosci 7:617-627.

Zheng B, Ho C, Li S, Keirstead H, Steward O, Tessier-Lavigne M (2003) Lack of enhanced spinal regeneration in Nogo-deficient mice. Neuron 38:213-224.

Zheng B, Lee JK, Xie F (2006) Genetic mouse models for studying inhibitors of spinal axon regeneration. Trends Neurosci 29:640-646. 